\title{
调控基因表达的 miRNA
}

\author{
陈 芳® 殷勤伟 $\mathbb{\oplus}^{*}$ \\ (1)中国科学院生物物理研究所, 系统生物学研究中心, 生物大分子国家重点实验室, 北京 100101; (2)中国科学院研究生院, \\ 北京 100049.*联系人, E-mail: jqwyin@sun5.ibp.ac.cn)
}

\begin{abstract}
摘要 近年来, 在小分子 RNA 中发现了一类与调节基因表达密切相关的分子 micro RNA (miRNA). miRNA 由内源性基因编码，可通过诱导 mRNA 的切割降解，翻译抑制或者其他形式的调节机制抑制靶 基因的表达. 寻找这类调节分子及其靶基因已成为研究的热点. 我们试图回顾 miRNA 的研究历史, 简 介 miRNA 分子的检测方法, 介绍 miRNA 在基因组中的位置、miRNA 靶基因的搜寻、miRNA 生物功 能的鉴定, 并探讨 miRNA 的作用机制, 最后展望 miRNA 在调控生命体的发生、生长、发育和分化等 方面的重要作用. 可以预测, 全部 miRNA 基因功能的鉴定必将给人们对生命体的研究带来新的理念、 方法和思路.
\end{abstract}

关键词 miRNA RNA 干扰 生物信息学 基因调控

micro RNAs (miRNAs) 是一类长约 22 核苷酸的非 编码的单链RNA分子, 它们广泛存在于从植物、线虫 到人类的细胞中 ${ }^{[1]}$. 最早发现的是 lin-4 和它的靶 mRNA, 即lin-14 $4^{[2]} .1993$ 年, Lee等人 ${ }^{[2]}$ 用经典的定位 克隆的方法在线虫(C. elegans)中克隆了lin-4 基因, 并通过定点突变发现lin-4 并不编码蛋白, 而是产生 一种小RNA分子. 这种小RNA分子能以不完全互补 的方式与其靶 mRNA的 3'非翻译端的特定区域相互 作用来抑制lin-14 的表达, 最终导致lin-14 蛋白质合 成的减少, 这种现象叫做转译抑制. 通过转译抑制, lin-4 控制着 C. elegans幼虫由L1 期向 L2 期的转化. 为 什么一个只有 $22 \mathrm{nt}$ 的RNA分子起着如此重要的调节 作用? 当时人们无法解释, 只能认为是一种稀少的 个别现象. 但是, 2000 年第 2 个miRNA let- $7^{[3]}$ 及其人 类和果蝇中同源物的发现改变了人们的看法, miRNA可能是一类进化上保守的、在生命中起着重 要调控作用的分子. 它们能有效地抑制相关蛋白质 的合成, 导致靶mRNA的降解, 或者其他形式的调节 机制来抑制靶基因的表达, 产生基因沉默 ${ }^{[4,5]}$. 近年 来发现miRNA 可能在基因表达调控领域中起着超乎 想象的重要作用, miRNA 序列、结构、丰度和表达 方式的多样性,使其可能作为蛋白质编码 $\mathrm{mRNA}$ 的强 有力的调节子. miRNA 的发现丰富了人们对蛋白质 合成控制的认识, 补充了在RNA水平对靶mRNA分 子进行更迅速和有效的调节，展现了细胞内基因表 达调控全方位多层次的网络系统 ${ }^{[6]}$. miRNA的发现也 是对中心法则中 RNA次要的中介角色的重要补充,
它将促使生物学家重新思考细胞遗传调控及其发育 等方面的重要问题.

\section{1 miRNA 的产生}

迄今为止, 在植物、线虫、果蝇和哺乳动物中已 发现了一千多个miRNA基因(表 1). 这些miRNA基因 首先在细胞核内转录成前体转录本 (pri-miRNA), 并 被加工成前体pre-miRNA, 然后被转运到细胞质被加 工成成熟的miRNA(图 1). Lee等人 ${ }^{[7]}$ 第一次证明一大 类miRNA由多聚酶 II (Pol II) 转录, 而且一些miRNA 的转录本(如let-7)具有 $5^{\prime}$ 帽和 $3^{\prime}$ poly(A)的结构, Pol II 的抑制剂能够抑制pri-miRNA的积累, 而Pol II 能 与miRNA的启动子免疫共沉淀. Rodriguez等人 ${ }^{[8]}$ 在基 因组水平对 miRNA的转录也进行了分析, 哺乳动物 中由内含子编码的miRNA, 其转录酶应该是 Pol II, Rodriguez等人 ${ }^{[8]}$ 检测的miRNA与 90 个蛋白编码基因 有关. 但是并不能排除miRNA还有其他多聚酶, 寻 找与miRNA相关的其他聚合酶还有待进一步的研究. Pri-miRNA在核内被RNase III 核酸酶 Drosha加工成 长约 $70 \mathrm{nt}$ 的发夹状的pre-miRNA ${ }^{[9]}$. 最近的报道指出 [10,11]，Drosha和另外的一些成分(如人类中的DGCR8 蛋白、果蝇中的Pasha蛋白)构成一个微小RNA处理器 (microprocessor)的复合体, pri- miRNA在这个 microprocessor中被加工成pre-miRNA. 关于在 pri-miRNA之前miRNA基因的转录本是如何加工的, 人们所知甚少. 线虫miRNA基因let-7 前体是一个相 对较长,, 0 且具有 poly (A)结构, Bracht等人 


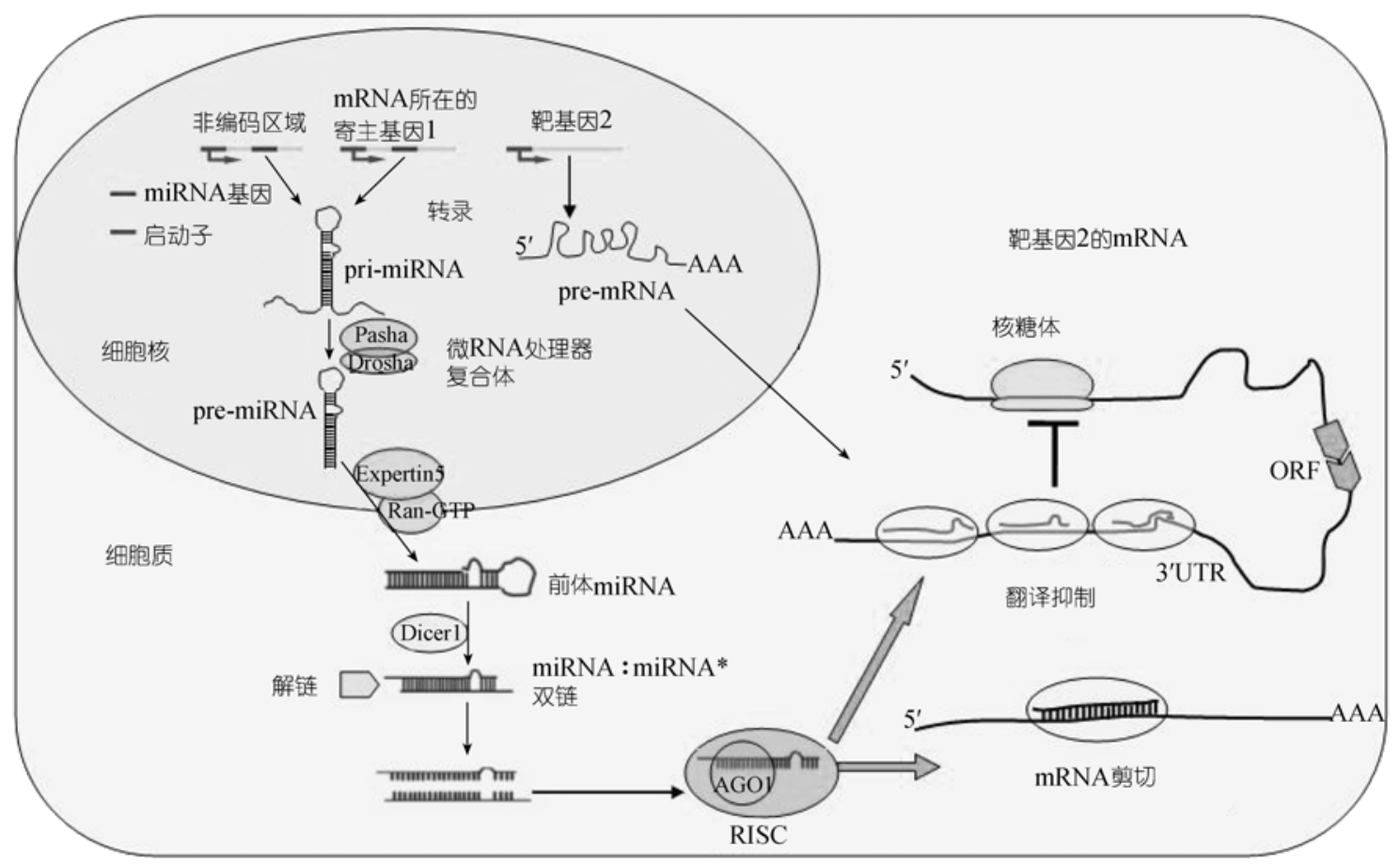

图 1 miRNA 基因的发生和作用机制

表 1 最新的已确认miRNA数量 $(\text { 来自 Rfam, 经实验数据证明 })^{[56]}$

\begin{tabular}{|c|c|c|c|}
\hline 物种 & 数量 & 物种 & 数量 \\
\hline 黑腹果蝇(D. melanogaster) & 78 & 非洲爪蟾(X. laevis) & 7 \\
\hline 拟暗果蝇(D.pseudoobscura) & 73 & 斑马鿕(D. rerio) & 33 \\
\hline 冈比亚按蚊(A. gambiae) & 38 & 拟南芥(A. thaliana) & 114 \\
\hline 意蜂(A. mellifera) & 25 & 栽培稻(O. sativa) & 173 \\
\hline 秀丽隐杆线虫(C. elegans) & 116 & 两色蜀㯟(S. bicolor) & 64 \\
\hline 桅杆线虫(C. briggsae) & 79 & 玉蜀㯟(Z. mays) & 40 \\
\hline 茶花鸡(G.gallus) & 122 & EB 病毒(Epstein Barr virus) & 5 \\
\hline 人(H. sapiens) & 227 & 卡波西肉瘤相关疮疹病毒 & 11 \\
\hline 小鼠(M. musculus) & 230 & (Kaposi sarcoma-associated herpesvirus) & \\
\hline 大鼠( $(R$. norvegicus $)$ & 191 & 人巨细胞病毒(H. cytomegalovirus) & 9 \\
\hline 狗(C. familiaris) & 6 & 鼠 $\gamma$ 疮疹病毒 68 (M. gammaherpesvirus 68) & 9 \\
\hline
\end{tabular}

发现let-7 转录本经历一个反式剪辑(trans-splicing)的 过程，生成一个叫做SL1RNA(spliced leader1)的中间 体. pre-miRNA在Exportin5 的帮助下从核进入胞质内 ${ }^{[13 \sim 15]}$, 这个过程还需要一个 $\mathrm{G}$ 蛋白因子 Ran参与 ${ }^{[13]}$. 能与exportin 5 结合的pre-miRNA必须具有大于 $16 \mathrm{nt}$ 配对的茎和 3 末端有 2 个悬垂碱基的结构特点, 末端 如果是 5 悬垂碱基将会抑制exportin5 对它的转运 $[15]$. 胞质中 pre-miRNA在Dicer酶的作用下, 被切割成双 链的miRNA：miRNA“配对分子, 然后成熟的miRNA 分子被解链, 单链的 miRNA进入侅糖蛋白复合
体miRNP(也叫RISC), miRNA通过与靶基因的 3'UTR 区互补配对, 指导miRNP复合体对靶基因mRNA进行 切割或者翻译抑制 $116 \sim 18]$.

\section{2 寻找 miRNA 基因}

\section{1 克隆方法寻找 miRNA 基因}

2001 年 miRNA 研究开始飞速发展. LagosQuintana等人 ${ }^{[19]}$ 设计出一种专门克隆小片段RNA的 方法(图 1(a)), 在 $2 \mathrm{~h}$ 的果蝇胚胎的裂解液里和Hela细 胞的总RNA中克隆了 37 个新的miRNA基因. Lau等人 
[20]用稍微改进的克隆方法在线虫中找到了 55 个 miRNA基因. Lee等人 ${ }^{[21]}$ 运用生物信息学和cDNA文 库相结合的方法找到了 13 个miRNA. 随后掀起了寻 找miRNA基因的热潮. Lagos-Quintana等人 20,23$]$ 继续 他们寻找miRNA基因的工作, 2002 年和 2003 年用同 样的方法分别在小鼠和人特异组织中克隆了 34 和 31 个新的miRNA, 并提出由于在克隆中已知的miRNA 重复出现和rRNA的干扰，用同样的克隆方法找到新 的 miRNA会越来越难, 这与后来 $\mathrm{Lim}$ 等人 ${ }^{[24,25]}$ 用 MiRscan计算机程序估计人类中只剩下 40 个未知 miRNA基因的结论一致. 近来，寻找miRNA的方法 越来越成熟, Ambros等人 ${ }^{[26]}$ 对克隆寻找miRNA的方 法进行了总结.

继而，人们将寻找miRNA基因的目光转向特异 的组织和发育的特异阶段 [27 31]. Mourelatos等人 ${ }^{[32]}$ 用 小片段克隆的方法在HeLa细胞中克隆了 31 个 miRNA, Houbavity等人 ${ }^{[29]}$ 在小鼠 ES 细胞中克隆了 15 个 miRNA，Dostie等人 ${ }^{[28]}$ 和 $\mathrm{Kim}$ 等人 ${ }^{[30]}$ 在哺乳动物神 经细胞中分别找到了 53 和 40 个miRNA, Ambros等人 ${ }^{[33]}$ 在线虫中又找到 21 个miRNA，Aravin等人 ${ }^{[27]}$ 在果 蝇的不同发育阶段找到了 63 个miRNA, Wang等人 ${ }^{[34]}$ 人在籼稻中找到了 20 个miRNA基因. Pfeffer等人 ${ }^{[35]}$ 在EB病毒的基因组中, 找到 5 个编码miRNA的基因, 这为 miRNA基因家族添加了病毒中的成员. 最近研 究人员又在另 3 种病毒中找到了 29 个病毒miRNA ${ }^{[36]}$. Suh等人 ${ }^{[37]}$ 在人胚胎干细胞中找到了 36 个miRNA基 因, 这些miRNA随着胚胎的发育表达量逐渐减少. Seitz等人 ${ }^{[38]}$ 在人的 14q32 区域找到 46 个miRNA基因, 发现它们多数在人的胚胎中表达, 只由母性染色体 表达, 并且受到上游 $200 \mathrm{~kb}$ 处的一个甲基化区域 (IG-DMR)调节. Kasashima等人 ${ }^{[39]}$ 运用克隆的方法检 测了 TPA诱导人白血病细胞HL-60 分化前后miRNA表 达谱的变化, 找到了 3 个新的miRNA, 并发现不同 miRNA在分化前后表达量不同. 现在科学家们已在不 同的种属中克隆了大量的新miRNA基因.

\section{2 检测 miRNA 表达的方法}

检测 miRNA最常用和直接的方法是 Northern杂 交，除此之外，还有RT-PCR，液相杂交和基因芯片技 术等. Lee等人 ${ }^{[7]}$ 通过Drosha-siRNA下调Drosha，抑制 pri-miRNA的加工, 再用RT-PCR的方法检测 miRNA 的表达. Sempere等人 ${ }^{[40]}$ 通过Northern 杂交的方法对 已知的 119 个miRNA基因的表达做了检测, 发现有一
些是在特异的组织表达, 有一些只是在不同组织的 表达量不一样, 而在脑组织表达的miRNA的作用方 式表明这些miRNA与脑神经元的分化相关. Allawi等 人 ${ }^{[41]}$ 将苂光方法引入 miRNA的研究中, 设计出一种 新的方法叫“Modified Invader”分析, 可快速而灵敏 地分析miRNA, 能从 50 100 ng总RNA或者是 1000 个细胞的裂解液中检测到目的miRNA.

miRNA基因芯片技术是一种更理想的快速有效 的检测miRNA表达图谱的方法. 这一方法被Liu等人 [42]首次报道用来检测 miRNA在不同肿瘤细胞中的表 达图谱, 245 个哺乳动物的miRNA被检测了, 结果的 重复性很好, 而且被Northern杂交、RT-PCR所证实. 之后, Esau等人 ${ }^{[43]}$ 运用miRNA芯片技术，检测细胞内 的miRNA表达图谱, 并且找到了 miR-143 和它的靶基 因. Calin等人 ${ }^{[44]}$ 通过miRNA芯片技术, 比较了哺乳 动物中 B细胞淋巴瘤和正常细胞中 miRNA的表达图 谱, 为 miRNA在肿瘤临床应用中, 提出了新的思路. Miska等人 ${ }^{[45]}$ 运用 miRNA芯片检测了小鼠脑发育中 138 个 miRNA基因的表达图谱. 在芯片的基础上, Nelson等人 ${ }^{[46]}$ 发展出一种叫做RAKE (RNA-primed, array-based Klenow enzyme assay)的方法能够在特定 的细胞和肿瘤中同时检测所有的miRNA的表达图谱.

原位杂交技术 ${ }^{[47,48]}$ 用来检测 miRNA, 更直观的 展示出 miRNA的表达方式, 是了解miRNA的时空表 达谱更方便的方法. Johnson等人 ${ }^{[49]}$ 通过在let-7 启动 子后加 GFP基因来跟踪检测线虫中let-7 的时空表达. Mansfield等人 ${ }^{[50]}$ 根据miRNA的作用机制设计出一种 带Laz报告基因的检测方法，被叫做“miRNA sensor”, 能特异的从原位检测到miRNA的表达.

\section{3 计算机软件预测 miRNA 基因}

miRNA 是由一个约 $70 \mathrm{nt}$ 的茎环结构前体而来, 并且在进化上是保守的, 因此可用计算机的方法来 识别.

MiRscan ${ }^{[24,25]}$ 是一个能特异的识别 2 个物种间的 同源序列的程序. Lim等人 ${ }^{[25]}$ 用它在C. elegans和 C. briggsae中寻找到同源的发卡结构, 通过已知miRNA 的训练后, 它去给那些发卡结构片段打分, 以预测线 虫中的miRNA. Lim等人 ${ }^{[24]}$ 用同样的方法, 在脊椎动 物里寻找miRNA基因, 并预测出人的miRNA基因数 在 200 255 之间, 这个数字大约是人类基因组的 $1 \%$. 2004 年, MiRscan程序又被用来检测了线虫中 miRNA 基因上游序列的同源性 ${ }^{[51]}$ ，和内含子中产生 
miRNA的寄主基因的同源性(host gene)，并且找到了 一个非常一致的序列模块, 运用这个不断改进的 MiRscan, 又在线虫中发现了 9 个miRNA, 并被PCR 实验所证实 ${ }^{[51]}$.

MiRseeker ${ }^{[52]}$ 是根据miRNA的 3 个特点开发出来 的: (i ) miRNA的保守性, 需要形成一个 70 100 nt的 前体; (ii) 在相似的物种中, miRNA是很保守的; (iii) 在相距较远的物种间, miRNA是有一定的分歧的. 它 包括以下 3 个步骤: (i) 通过AVID寻找果蝇中基因 间的保守的序列; (ii) 通过mfold辨认此序列是否能 形成保守的茎环结构, 并给这个结构打分评价; (iii) 评价 miRNA在不同物种中的分歧模式. 最后, miRseeker也需要通过生物化学的方法, 加以验证.

Legendre等人 ${ }^{[53]}$ 用“ERPIN”代替“blast”从基因组 中搜寻与已知miRNA类似的miRNA基因, 输入已知 miRNA软件可以根据已知miRNA的结构从基因组中 搜寻到结构类似的候选miRNA基因.

除了专门用于寻找miRNA基因的程序和软件外, 象 mfold 和 Srnaloop 是 RNA折叠中常用的软件. Hofacher 等人 ${ }^{[54]}$ 报道在维也纳 RNA 网站 (http: //rna.tbi.univie.ac.at/)提供了一个界面能够调用多个 常用的RNA二级结构预测软件.

\section{4 miRNA 的命名及 $\mathrm{Rfam}$ 数据库}

miRNA种类越来越多, 而且在研究中发现, 很 多 miRNA是同源的, 为了防止 miRNA的混淆, 最初 的研究者就给发现的miRNA作了命名规则 ${ }^{[19]}$ :

（i ） miRNA 简写成 miR-No., 它的基因简写成 mir-No.;

(ii ) 高度同源的 miRNA 在 No.其后加英文字母 (小写，一般从 a 开始); （iii）多基因拷贝的再在后面加 -No. （如 miR-2a-1).

之后人们又对命名规则作了补充 ${ }^{[55,56]}$. 不同物 种中同源的miRNA最好用同一个名字; 如果一个前 体的 2 个臂分别产生 1 个miRNA, 则根据克隆实验, 看看哪个是主要的成熟产物, 次要的在后面加“*”号, 如miR-56 和miR-56*(*表示量少的); 如果不能区分 表达量的多少, 可以如下表达, 如: miR-142-5p(表示 在 5'端的臂, 以前有人用 miR-142-s) 和 miR-142-3p (表示 3'端的臂, 以前有人用miR-142-as). 在Rfam数 据库中, 在每个 miRNA名称前加上了物种的名称, 如：hsa-miR-138. 植物中 miRNA的基因用斜体表示, 如MIR156.

为了更好地管理 miRNA, 方便人们查询, 研究者 们建立了 miRNA 的数据库 http: //www.sanger.ac.uk/ Software/Rfam/mirna/. 现在这个数据库已经更新到 了 6.0 版本(2005 年 4 月), 共有 1650 个 miRNA(表 1). Rfam 只有在文章发表后才给新发现的 miRNA 一个 确定的名字, 在发表前作者可以用一个临时的名字 命名送审.

\section{3 miRNA 的靶基因}

虽然现在已经找到了过千个miRNA, 并提出它 们在细胞增殖、分化、代谢与死亡中发挥着重要的调 节作用, 但是至今为止, 真正确认功能的miRNA还 是微乎其微 $[1,57,58]$. 从 1993 年发现第 1 个miRNA到现 在, 在动物中发现的确认功能的miRNA不超过 10 个 ${ }^{[59 \sim 67]}$ (表 2).

\section{1 基因篮选和定位克隆寻找 miRNA 靶基因}

通观科学家们寻找 miRNA 及其靶基因的方法发 现，现在已知靶基因的 miRNA 多是通过传统的基因

表 2 已知功能的 miRNA 基因

\begin{tabular}{|c|c|c|c|c|}
\hline miRNA & 物种 & 靶基因 & 生物学作用 & 文献 \\
\hline Lin-4 & 线虫 & Lin-14 和 lin-28 & 时序发育 & {$[2]$} \\
\hline Let-7 & 线虫 & Lin-41 和 hbl-1 & 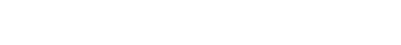 & {$[3]$} \\
\hline lsy-6 & 线虫 & $\operatorname{Cog}-1$ 转录因子 & 调节化学感受器的左右不对称性 & [61] \\
\hline $\operatorname{miR}-273$ & 线虫 & die-1 转录因子 & 调节化学感受器的左右不对称性 & {$[64]$} \\
\hline Bantam & 果蝇 & Hid 前调亡基因 & 发育中调节细胞增殖与凋亡 & {$[59,60]$} \\
\hline $\operatorname{miR}-14$ & 果蝇 & 未知 & 调亡和脂类代谢 & {$[63]$} \\
\hline $\operatorname{miR}-181$ & 哺乳动物 & 未知 & 造血系统的分化 & {$[62]$} \\
\hline miR-196 & 哺乳动物 & HОХВ8 & 脊椎动物发育 & {$[50,65]$} \\
\hline miR-143 & 哺乳动物 & ERK5 & 脂肪细胞的分化 & [43] \\
\hline $\operatorname{miR}-375$ & 哺乳动物 & Mtpn & 胰岛素分泌的调节 & [66] \\
\hline $\operatorname{miR}-430$ & 斑马鱼 & 待定 & 脑的形态发生 & [67] \\
\hline
\end{tabular}


篮选(gene screen)和定位克隆的方法发现的 ${ }^{[2,3,59 ~ 61]}$ (图 2). Brennecke等人 $\frac{59,60]}{4} \mathbb{l}^{2}$ 现bantam 基因及其靶基 因hid, 包括典型的如下几个过程: ( i ) Bantam基因表 达miRNA的证据: (1) 通过插入突变, 找到bantam这 个位点, 能够促进组织过度生长; (2) bantam缺陷的 个体, 具有生长缺陷或者是蛹化期致死; (3) 在 2 个 物种间比对(在Drosophila与Anopheles用Clustal W程 序比对), 发现一个约 $90 \mathrm{nt}$ 的区域非常相似, 有 30/31 $\mathrm{nt}$ 是相同的, 再用mfold进行预测发现 2 个物种内的 这段区域能形成相似的稳定的发卡结构; (4) 用这 $31 \mathrm{nt}$ 作探针, 通过Northern 杂交在野生和 Gal4 诱导 表达的L3 期中找到这个 $21 \mathrm{nt}$ RNA和它的更大的前 体. (ii ) 证明hid是bantam的靶基因的过程：(1) 用计 算机预测的方法找到前调亡基因hid的 3' UTR区，有 3 个能与bantam作用的靶点, 而且直接观察发现还有 2 个靶点; (2) 对比这些 3' UTR区在 2 个物种的同源 性(D. pseudoobscura与D. melanogaster), 发现它们高 度同源；（3）表达报告基因tubulin-EGFP，在它的末尾 连上hid的 $3^{\prime}$ UTR区的靶点, 同时转入EP表达的 bantam, 发现GFP苂光明显下降, 而且 5 个靶
点同时存在的效果要比单个的好; (4) 同时表达 bantam和hid, 通过原位杂交, 能检测内源表达的hid 也能被bantam抑制. 根据一个miRNA可能调节多个 靶基因的观点 ${ }^{[1]}$, 发现bantam能够促进细胞的增殖, 很可能bantam还具有一个细胞增殖负调控的靶基因.

Chen等人 ${ }^{[62]}$ 的方法略有不同. 他们先用miRNA 的cDNA克隆的方法在小鼠骨髓中克隆了 100 多个 miRNA, 并且特别的研究了其中 3 个的功能. 结果发 现在造血干细胞中异位表达 miR-181 能够促进造血 干细胞的定向分化, 但是没有找到它的靶基因. $\mathrm{Xu}$ 等 人 ${ }^{[63]}$ 通过P插入元件找到突变子, 发现具有死亡抑制 的功能, 并证明编码miR-14. 通过功能缺失和异位表 达的方法, 证明miR-14 有着抑制reaper导致的细胞死 亡和参与脂肪代谢的功能. 实验提出 miR-14 可能的 靶点是Drice, 但没有直接的证据证明.

\section{2 计算机程序对靶基因的预测}

从已有的资料看来，传统的方法寻找 miRNA 的 靶基因比较艰难, 由于目标不明确, 效率较低. 研究 人员想到用生物信息学的方法来取代大量的分子克 隆的工作, 并且获得很好的目的性.

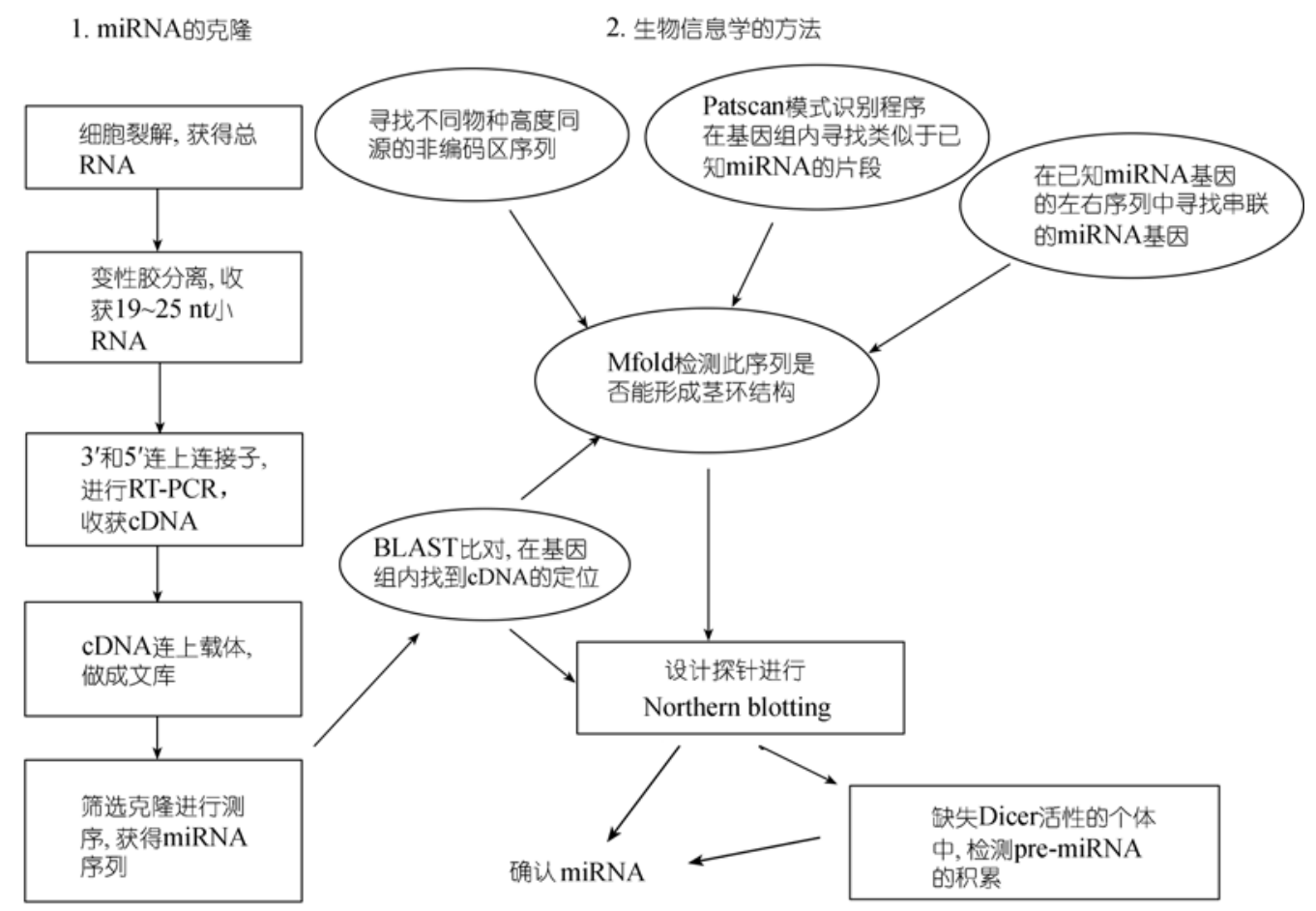

图 $2 \mathrm{cDNA}$ 克隆与生物信息学相结合的方法寻找 miRNA 基因 方形框为分子生物学方法, 椭圆形框为生物信息学方法; 每条路线都可以确定一个 miRNA 


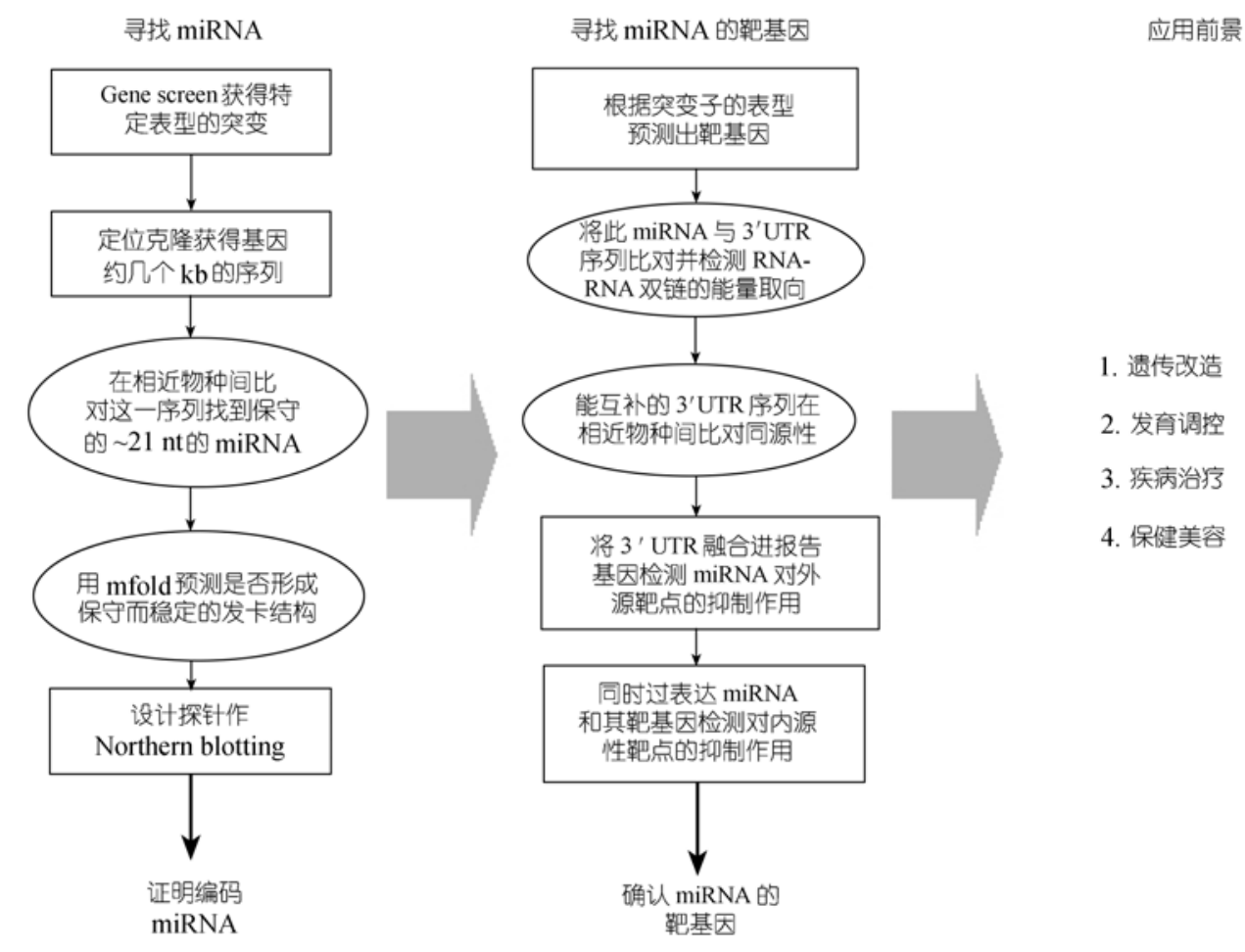

图 3 miRNA 功能解析

方形框为分子生物学方法, 椭圆形框为生物信息学方法

Stark等人 ${ }^{[68]}$ 的方法是首先构建一个在 D. melanogaster和D. pseadoobscura中保守的 $3^{\prime}$ UTR数据库, 能 覆盖D. melanogaster三分之二的基因. 用HMMer比 对程序, 找到能与miRNA前 8 个碱基配对的 3' UTR 靶序列; 然后再用mfold对整个miRNA：mRNA靶序 列进行动力学检测, 并给出 $\Delta G$ 值; 最后计算 $Z$ 值, 取 $Z \geq 3$ 作为篮选靶序列的阈值. 通过这一程序成功的 在数据库里找到 5 个已知的miRNA和它们的靶基因, 同时预测出 3 个新的miRNA和它们的靶基因: miR-7 和Notch, miR-2 和前凋亡基因、miR-277 和代谢酶类 (但随后的生物学实验证据并不强). 结果可在 http://www.russell.embl.de/miRNAs查询.

Enright等人 ${ }^{[69]}$ 开发出一种计算机预测的方法: 先从D. melanogaster和D. pseadoobscura中通过blastn 和AVID比对构建出 $3^{\prime}$ UTR序列库; 然后用MiRanda 算法评价, MiRanda是一个类似于Smith-Whatman的 算法, 但它是用来检测互补配对的(其中允许 $G$ ：U配 对), 此外这一算法还加入了RNA二级结构预测的程 序 (来源于 RNAlib), 能够从动力学角度预测 miRNA：靶mRNA的稳定性; 再将前面的结果在 3 种
生物中检验保守性 (D. melanogaster, D. pseudoobscura和 A. gambiae), 最后给出 $S$ 值和 $\Delta G$ 值. 这个方法 考虑了以下几个特点: ( i ) 局部位置的互补比对; (ii) RNA-RNA双链自由能的预测; (iii) 靶位点的保守性. 结果显示, 靶基因多是发育阶段特异表达的、与细胞 分化、形态、发育协调相关的基因, 而且有很多是转 录因子. 其中, 作者提出 $3^{\prime}$ UTR调节很类似于转录中 的 $5^{\prime}$ promoter的调节, 这说明在这 2 个机制之间可能 有一定的联系. 结果可在http:// www.microrna.org查 询.

Lewis等人 ${ }^{[70]}$ 开发了一个计算机预测的方法 - TargetScan法, 用来预测哺乳动物内 miRNA的靶 基因. 这一方法基于 2 点考虑: ( i ) 考虑miRNA: mRNA(靶向基因mRNA片段)双链结构的稳定性; (ii) 检测这些靶向基因mRNA的 3' UTR区在不同物种间 的保守性. 此搜索方法重点强调了 miRNA的第 2 8 个碱基, 将这段序列作为搜索的“种子序列”, 必须严 格配对; 然后用RNAfold 程序向两边延展寻找配对 的序列; 通过RNAeval给出自由能 $G$ 值; 最后给出 $Z$ 值 和 $R$ 值, Z值越大, $R$ 值越小, 靶向的可能性就越大. 通 
过对在人、小鼠、大鼠中保守的miRNA的搜索, 找到 了 451 个可能的miRNA靶向序列; 通过对人、小鼠、 大鼠、河豚 4 种动物中保守的miRNA搜索, 找到了 115 个可能的靶向序列. 结果可在网上查询 http:// genes.mit.edu/targetscan.

Kiriakidou等人 ${ }^{[71]}$ 开发一个“DIANA-microT”的 程序, 通过计算机的方法来预测哺乳动物中 miRNA 的靶基因. Rehmsmeier 等人 ${ }^{[72]}$ 开发了一个 “RNA-hybrid”的程序，来预测果蝇的miRNA的靶基 因, 他们的信息公布在互联网上(http://bibiserv. techfak.uni-bielefeld.de/rnahybrid/). PicTar是由Krek等人 ${ }^{[73]}$ 开发的预测 miRNA靶基因的软件，他们搜索了 8 个脊椎动物物种的基因组和现有的miRNA，它对靶 基因预测具有较高的准确性.

John等人 ${ }^{[74]}$ 运用miRanda软件预测了 218 个哺乳 动物的miRNA基因的靶基因, 发现有 2000 个的哺乳 动物的基因有着保守的 $3^{\prime}$ UTR靶点, 这些蛋白多数 涉及转录因子、miRNA机制本身相关的蛋白、泛素 机制相关的蛋白, 这些可能的靶蛋白说明miRNA的 作用构建了一个反馈调控网络，并且根据他们的预 测, 占基因组数量 1\%的miRNA基因可能调控着 $10 \%$ 的基因组蛋白. 预测软件在互联网上 (http:// www.microrna.org).

综合人们用计算机的方法预测 miRNA 的工作, 发现各种计算方法都具有如下的特点: ( i ) miRNA 及其靶向序列的保守性; (ii ) miRNA：靶 mRNA 配 对中 5端非常重要, 其他位置允许 $G$ ：U 配对; (iii)
miRNA 与靶向基因可能是一对多的关系. 通过计算 机预测出的 miRNA 靶基因, 如表 3 所示.

\section{4 miRNA 的作用机制及功能}

\section{1 miRNA 的作用机制}

miRNA 转录前体 (Pri-miRNA) 首先在核内被 Rnase III 核酸酶 Drosha加工成约 70 个核苷酸长的发 夹状的RNA, 在Exportin5 的帮助下从核内进入胞质 内, 然后在Dicer酶的作用下, 单链的miRNA进入一

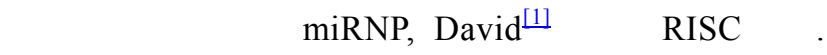
miRNA通过与靶基因的 3' UTR区互补配对, 指导 miRNP复合体对靶基因 mRNA进行切割或者翻译抑 制 ${ }^{[18-20]}$. MiRNA到底是抑制还是切割取决于miRNA 与靶序列互补配对的程度, 互补配对高的可能进行 切割, 而配对低的就只是抑制. 植物的 miRNA与靶 基因配对程度高多数是进行切割, 而动物中 miRNA 与靶序列的配对性不好, 多数进行翻译抑制.

线虫中, lin-4 RNA并不降低lin-14 和lin-28 的 mRNA水平, 但能降低它们的蛋白水平 ${ }^{[2]}$. Let-7 也通 过与lin-41 的 3' UTR区配对, 来抑制mRNA的表达, 并不进行切割 ${ }^{[3]}$. 现在普遍认为, 细胞中的miRNA能 与靶基因mRNA的 3'UTR部分配对, 指导miRNP对 其翻译的抑制. 这与现在所知的真核基因的翻 译 调控主要是在 3' UTR区域的思想一致 ${ }^{[75]}$, 最近 $X i e$ 等人 ${ }^{[76]}$ 在人、小鼠、大鼠和狗的基因组比对分析 指出, 在 3' UTR区域存在大量的调节结构域, 而近一 半的这些调节结构域与 miRNA的调节有关. 其中 miRNA的 5'端的 2 8 个核苷酸对于miRNA与 $3^{\prime}$ UTR

表 3 预测的 miRNA 及其可能的靶基因

\begin{tabular}{|c|c|c|c|c|}
\hline miRNA & 物种 & 靶基因 & 生物学功能 & 文献 \\
\hline$m i R-7$ & 果蝇 & HLHm3, hairy 类的 HLH 转录因子, $m 4$ Brd 家族 & Notch 介导的神经分化 & [68] \\
\hline $\operatorname{miR}-14$ & 果蝇 & grim, reaper, sickle & 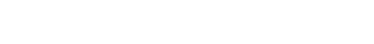 & {$[68]$} \\
\hline \multirow[t]{2}{*}{$M i R-1$} & 人类 & $B D N F$ & 生长因子神经发育 & [70] \\
\hline & & $G 6 P D$ & 抗氧化性 & \\
\hline$M i R-19 a$ & 人类 & PTEN & 肿瘤抑制基因 & [70] \\
\hline \multirow[t]{2}{*}{$M i R-23 a$} & 人类 & $S D F-1$ & 造血前体细胞的生长和定位 & [70] \\
\hline & & $B R N-3 b$ POU-domain 转录因子 & 神经发育 & \\
\hline$M i R-26 a$ & 人类 & $S M A D-1$ & TGF- $\beta$ 通路 & [70] \\
\hline \multirow[t]{2}{*}{$M i R-34$} & 人类 & Delta-1 跨膜蛋白 & 激活 Notch & [70] \\
\hline & & Delta 的 Notch 1 跨膜受体 & 在分化中决定细胞的命运 & \\
\hline \multirow[t]{2}{*}{$M i R-101$} & 人类 & ENX-1 polycomb 基因 & 造血细胞的增殖 & [70] \\
\hline & & $N-M Y C$ 类的 HLH 转录因子 & 前癌基因, 细胞的分化和增殖 & \\
\hline MiR-130 & 人类 & MCSF & 单核吞噬细胞的调节 & [70] \\
\hline
\end{tabular}


的配对非常重要. 首先, miRNA的 2 8 个核苷酸能很 好的与靶序列配对 ${ }^{[77]}$; 其次, 靶序列在不同物种中 通常保守 ${ }^{[68]}$; 再次, miRNA的 2 8 碱基在同源的 miRNA中保守 ${ }^{[70]}$. 因此，人们用此来预测 miRNA的 靶基因. miRNA抑制mRNA的翻译机制至今为止是个 迷 ${ }^{[1]}$. David ${ }^{[1]}$ 提出 2 种可能性: (i ) 在mRNA起始翻 译后, miRNA通过与 $3^{\prime}$ UTR 作用抑制翻译的进行, 这 样可能可以通过检测 mRNA上的核糖体的密度而加 以证实; (ii) mRNA的翻译并不被阻止, 但是翻译的 多肽不断的被miRNP复合体, 或者再结合别的蛋白 复合体切割, 当然这需要肽酶参与. 这些机制的研究 可以和 siRNA 的作用相结合起来，近来关于 Argonaute蛋白 PAZ结构域晶体结构的测定揭示了 RISC与RNA结合的作用机制 ${ }^{[78 \sim 80]}$. 当然miRNA抑制 机制的揭示有待于miRNA领域的深入研究.

\section{2 miRNA 的功能}

miRNA是一种广泛存在的对基因表达进行微调 的分子. 在人类基因组中 miRNA基因占据大约 $1 \%$ 的 数量, 虽然在不同的时空发育阶段, 它们的表达量不 同，但是多种miRNA在同一个细胞中的表达使得细 胞处在一种内在的miRNA环境中, 这个环境控制着 成千上万的编码基因的mRNA水平，使得各种蛋白的 表达处在一个适合的水平. Bartel等人 ${ }^{[57]}$ 将这些miRNA 比作微型电阻, 根据miRNA与靶基因互补的程度, 执 行从切割(100\%抑制)到微弱抑制的不同的抑制作用. 象lin-4 作用于lin14 和lin28; let-7 作用于lin41 和hbl-1, 起着明显的表型改变, 是在miRNA家族中为数较少 的, 更多的则是起着微调的作用. 像 miR-7 和miR-14 有着多个靶基因, 它们从一个整体上起着介导某一 功能的作用. 由于有多个靶点, 有些miRNA会有大 量的表达, 比如miR-2 在一个细胞内有约 50000 个拷 贝，这远多于普通mRNA的表达量．通过芯片技术 Lee等人 ${ }^{[81]}$ 研究了通过导入某些miRNA能够导致上 百个基因mRNA水平的下调，虽然并不能完全肯定所 有的这些下调基因是由miRNA直接导致, 但这给出 了直接证据证明一个miRNA可以调控多个靶基因. 另一些则是多个 miRNA作用于一个靶基因上, 而最 近开发的同时检测所有 miRNA的表达图谱的技术 ${ }^{[46]}$, 使研究者能更方便地从整体上掌握特定的时空阶段 miRNA产生的生物学效应. miRNA这种转录后微调 的优点有如下几点:

（i ）比从蛋白水平的调节更节省能量; (ii ) 相对于转录调节, miRNA 的效果更快而且 是可逆的;

(iii) 对于一些只需微量蛋白改变的现象, 只有 通过 miRNA 来达到. 比如对某种蛋白来说, 1 2 个 mRNA 拷贝就足以过量表达, 这时就需要 miRNA 来 调节控制蛋白的水平;

(iv) 内含子中编码的 miRNA 是一种细胞内资源 的高效利用.

就像前面提到的一样, miRNA主要通过抑制它的 靶基因来起调控作用, 至今为止没有发现有上调能力 的miRNA. 从表 2 和表 3 可以看出, miRNA的作用遍及 生命体的发生、生长、发育、分化和死亡的过程. Lewis 等人 ${ }^{[70]}$ 的预测结果发现, 预测的miRNA的靶基因多数 是参与转录、信号转导、肿瘤发生的基因. 虽然miRNA 的作用也是特异的, 特别是 5端的 2 8 个碱基, 特异 性的靶向与它的靶基因, 但是与 siRNA不同的是 miRNA的特异性并不是那么强, 在生物体内往往一个 miRNA作用于多个靶基因, 如lin-4 作用于lin14 和 lin2 $8^{[2]}$, let-7 作用于lin41 和 Hbl-1 $1^{[3]}$ (表 2), 这就是人们 提出的“多个靶点”的假说. 人们也提出可能多个 miRNA调控一个靶基因 ${ }^{[3,82]}$. 因此，miRNA的调控作 用很可能是一种调控网络, 它需要接受某些信号的刺 激, 从整体上调控有机体的生命活动.

miRNA的研究现在还处在理论水平上, 讨论关 于miRNA的应用还为时过早, 但miRNA作为体内正 常表达的基因, 是否与人类疾病 ${ }^{[83]}$ 和植物培育相关, 还有待进一步的研究. 以 miRNA 为靶点或者以 miRNA为治疗手段的设想 ${ }^{[83]}$, 以后可能会成为焦点. 而Calin等人 ${ }^{[44]}$ 报道的miRNA基因芯片技术也很可能成 为肿瘤诊断的工具. Suh等人 ${ }^{[37]}$ 报道, 在人胚胎干细胞 中表达的miRNA与其他细胞类型的miRNA有很大的不 同, miRNA作为人器官定向分化的调节子, 为以后人 器官体外培植带来促进作用. miRNA作用和功能的 揭示必定给miRNA的应用带来广阔前景.

\section{5 展望}

自从在四膜虫中发现核酶以来，引起了整个生 物学界对RNA及其作用的关注, 也让人们就生命起 源的问题争论了十几年. 在人们对生命由RNA起源 的争论降温的时候, 1998 年, Fire等人报道在线虫中 发现了 RNAi的现象, 重新又将人们的视线转到RNA 上来. RNAi是一个强大而特异的基因沉默机制, 可 成为研究基因功能 ${ }^{[84]}$ 和疾病治疗 ${ }^{[85]}$ 的有用工具, 这 
是生物研究领域里的一个突破性研究成果. miRNA 研究的飞速发展也得益与RNAi的发现. 但与此不同 的是, miRNA是生物体内源的小RNA分子, 研究表明 它们在生物体内不仅仅是代谢的产物, 而是机体有 目的编码的重要的调控分子. 它们参与生物体的生 长、发育、衰老、死亡的调控. 随着研究的深入, miRNA 将在生命起源和物种进化、基因表达调控的 复杂性、疾病发生和发展的机制等方面起到更为深远 的作用. 同时, miRNA的研究亦将为RNAi技术的应 用提供新的依据、思路和空间. 现在，欧美等发达国 家已将有关miRNA的研究广泛而深入地运用到组织 器官的定向发育, 细胞生长分化的时空调节, 信号通 路的开启和关闭, 细胞周期的监测与调控、学习与记 忆，肿瘤的逆分化、肥胖、衰老和死亡，疾病的防治 以及有目的的基因表达调控上.

寻找调控的 miRNA 基因以及 miRNA 的靶基因, 揭示 miRNA 具体的作用机制是非常重要的. 就目前 的研究来看, 虽然在各个物种中发现的 miRNA 数量 不少, 但是能给出直接证据证明 miRNA 的靶基因及 其功能的 miRNA 很少, 而且多数是通过篮选突变子 获得的, 这说明现在寻找 miRNA 基因的研究与它功 能的研究是脱节的, 也证明人们通过现有方法得出 的 miRNA 的一些观点可能存在问题(如人类 miRNA 上限为 255 个的报道). 现在摆在科学家们面前的两 大问题就是: 在各种生物中找齐这些 miRNA 和找到 它们的靶基因并揭示它们的功能, 这也可以说是后 基因组时代需要解决的问题之一. 当然结合 RNAi 和 生物信息学的方法会使 miRNA 功能的研究更简便, 而且有可能开辟一条共同的研究路线, 逐一找到 miRNA 的靶基因并揭示它们的功能. 由于 miRNA 基 因对于生物的生长, 发育, 分化非常重要, 而且具有 特异的时空表达的特点, 人们可以设计出 miRNA 的 检测芯片，在不同的生物、不同的发育时期、不同的 组织细胞内, 检测 miRNA 的表达图谱, 更精确地掌 握生命发展调控的过程. 全部 miRNA 基因功能的揭 示可能将会给人们对生命现象的理解带来一场新的 革命.

\section{参考文献}

1 David P B. MicroRNAs: Genomics, biogenesis, mechanism, and function. Cell, 2004, 116: 281 297[DOI]

2 Lee R C, Feinbaum R L, Ambros, V. The C.: elegans heterochronic gene lin-4 encodes small RNAs with antisense complementarity to lin-14. Cell, 1993, 75: 843 854[DOI]

3 Reinhart B J, Slack F J, Basson M, et al. The 21 nucleotide let-7 RNA regulates developmental timing in Caenorhabditis elegans. Nature, 2000, 403: 901 906[DOI]

4 Yin J Q, Wang Y. siRNA-mediated gene regulation system: Now and the future. Int J Mol Med, 2002, 10: 355 365

5 Tan F L, Yin J Q. Application of RNAi to cancer research and therapy. Front Biosci, 2005, 10: 1946 1960

6 Yin J Q, Jiang L. MiRNA-directed gene intertalk. Chin Acad Forum, 2004, 11(20): 1 4

7 Lee Y, Kim M, Han J, et al. MicroRNA genes are transcribed by RNA polymerase II . EMBO J, 2004, 23(20): 4051 4060[DOI]

8 Rodriguez A, Griffiths-Jones S, Ashurst J L, et al. Identification of mammalian microRNA host genes and transcription units. Genome Res, 2004, 14(10A): 1902 1910[DOI]

9 Lee Y, Ahn C, Han J, et al. The nuclear RNase III Drosha initiates microRNA processing. Nature, 2003, 425: 415 419[DOI]

10 Denli A M, Tops B B J, Plasterk R H A, et al. Processing of primary microRNAs by the microprocessor complex. Nature, 2004, 432: 231 235[DOI]

11 Gregory R I, Yan K, Amuthan G, et al. The microprocessor complex mediates the genesis of microRNAs. Nature, 2004, 432: 235 240[DOI]

12 Bracht J, Hunter S, Eachus R, et al. Trans-splicing and polyadenylation of let-7 microRNA primary transcripts. RNA, 2004, 10(10): 1586 1594[DOI]

13 Lund E, Guttinger S, Calado A, et al. Nuclear export of microRNA precursors. Science, 2004, 303: 95 98[DOI]

14 Kim V N. MicroRNA precursors in motion: Exportin-5 mediates their nuclear export. Trends Cell Biol, 2004, 14(4): 156 159[DOI]

15 Zeng Y, Cullen B R. Structural requirements for pre-microRNA binding and nuclear export by Exportin 5. Nucleic Acids Res, 2004, 32(16): 4776 4785[DOI]

16 Hutvágner G, Zamore P D. A microRNA in a multiple-turnover RNAi enzyme complex. Science, 2002, 297: 2056 2060[DOI]

17 Zeng Y, Yi R, Cullen B R. MicroRNAs and small interfering RNAs can inhibit mRNA expression by similar mechanisms. Proc Natl Acad Sci USA, 2003, 100: 9779 9784[DOI]

18 Doench J G, Peterson C P, Sharp P A. siRNAs can function as miRNAs. Genes Dev, 2003, 17: 438 442 [DOI]

19 Lagos-Quintana M, Rauhut R, Lendeckel W, et al. Identification of novel genes coding for small expressed RNAs. Science, 2001, 294: 853 858[DOI]

20 Lau N C, Lim L P, Weinstein E G, et al. An abundant class of tiny RNAs with probable regulatory roles in Caenorhabditis elegans. Science, 2001, 294: 858 862[DOI] 
21 Lee R C, Ambros V. An extensive class of small RNAs in Caenorhabditis elegans. Science, 2001, 294: 862 864[DOI]

22 Lagos-Quintana M, Rauhut R, Yalcin A, et al. Identification of tissue-specific microRNAs from mouse. Curr Biol, 2002, 12: $735 \sim 739[\mathrm{DOI}]$

23 Lagos-Quintana M, Rauhut R, Meyer J, et al. New microRNAs from mouse and human. RNA, 2003, 9: 175 179[DOI]

24 Lim L P, Glasner M E, Yekta S, et al. Vertebrate microRNA genes. Science, 2003, 299: 1540[DOI]

25 Lim L P, Lau N C, Weinstein E G, et al. The microRNAs of Caenorhabditis elegans. Genes Dev, 2003, 17: 991 1008[DOI]

26 Ambros V, Lee R C. Identification of microRNAs and other tiny noncoding RNAs by cDNA cloning. Methods Mol Biol, 2004, 265 : $131 \sim 158$

27 Aravin A A, Lagos-Quintana M, Yalcin A, et al. The small RNA profile during Drosophila melanogaster development. Dev Cell, 2003, 5: 337 350[DOI]

28 Dostie J, Mourelatos Z, Yang M, et al. Numerous microRNPs in neuronal cells containing novel microRNAs. RNA, 2003, 9: 180 186[DOI]

29 Houbaviy H B, Murray M F, Sharp P A. Embryonic stem cell-specific microRNAs. Dev Cell, 2003, 5: 351 358[DOI]

30 Kim J, Krichevsky A, Grad Y, et al. Identification of many microRNAs that copurify with polyribosomes in mammalian neurons. Proc Natl Acad Sci USA, 2004, 101: 360 365[DOI]

31 Michael M Z, O'Connor S M, van Holst Pellekaan N G, et al. Reduced accumulation of specific microRNAs in colorectal neoplasia. Mol Cancer Res, 2003, 1: 82 91

32 Mourelatos Z, Dostie J, Paushkin S, et al. miRNPs: A novel class of ribonucleoproteins containing numerous microRNAs. Genes Dev, 2002, 16: 720 728[DOI]

33 Ambros V, Lee R C, Lavanway A, et al. MicroRNAs and other tiny endogenous RNAs in C. elegans. Curr Biol, 2003, 13: $807 \sim 818[\mathrm{DOI}]$

34 Wang J F, Zhou H, Chen Y Q, et al. Identification of 20 microRNAs from Oryza sativa. Nucl Acids Res, 2004, 32(5): 1688 1695[DOI]

35 Pfeffer S, Zavolan M, Grasser F A, et al. Identification of virus-encoded microRNAs. Science, 2004, 304(5671): 734 736[DOI]

36 Pfeffer S, Sewer A, Lagos-Quintana M, et al. Identification of microRNAs of the herpesvirus family. Nat Methods, 2005, 2: 269 276[DOI]

37 Suh M R, Lee Y, Kim J Y, et al. Human embryonic stem cells express a unique set of microRNAs. Dev Biol, 2004, 270(2): 488 498[DOI]

38 Seitz H, Royo H, Bortolin M L, et al. A large imprinted microRNA gene cluster at the mouse Dlk1-Gt12 domain. Genome Res, 2004, 14(9): 1741 1748[DOI] https://engine.scichina.com/dgs/
39 Kasashima K, Nakamura Y, Kozu T. Altered expression profiles of microRNAs during TPA-induced differentiation of HL-60 cells. Biochem Biophys Res Commun, 2004, 322(2): 403 410[DOI]

40 Sempere L F, Freemantle S, Pitha-Rowe I, et al. Expression profiling of mammalian microRNAs uncovers a subset of brain-expressed microRNAs with possible roles in murine and human neuronal differentiation. Genome Biol, 2004, 5(3): R13[DOI]

41 Allawi H T, Dahlberg J E, Olson S, et al. Quantitation of microRNAs using a modified Invader assay. RNA, 2004, 10(7): 1153 1161[DOI]

42 Liu C G, Calin G A, Meloon B, et al. An oligonucleotide microchip for genome-wide microRNA profiling in human and mouse tissues. Proc Natl Acad Sci USA, 2004, 101(26): 9740 9744[DOI]

43 Esau C, Kang X, Peralta E, et al. MicroRNA-143 regulates adipocyte differentiation. J Biol Chem, 2004, 279(50): 52361 52365[DOI]

44 Calin G A, Liu C G, Sevignani C, et al. MicroRNA profiling reveals distinct signatures in B cell chronic lymphocytic leukemias. Proc Natl Acad Sci USA, 2004, 101(32): 11755 11760[DOI]

45 Miska EA, Alvarez-Saavedra E, Townsend M, et al. Microarray analysis of microRNA expression in the developing mammalian brain. Genome Biol, 2004, 5(9): R68[DOI]

46 Nelson P T, Baldwin D A, Scearce L M, et al. Microarray-based, high-throughput gene expression profiling of microRNAs. Nat Methods, 2004, 1: 155 161[DOI]

47 Chen X. A microRNA as a translational repressor of APETALA2 in Arabidopsis flower development. Science, 2004, 303: 2022 2025[DOI]

48 Juarez M T, Kui J S, Thomas J, et al. MicroRNA-mediated repression of rolled leaf1 specifies maize leaf polarity. Nature, 2004, 428(6978): 84 88[DOI]

49 Johnson S M, Lin S Y, Slack F J. The time of appearance of the $C$. elegans let-7 microRNA is transcriptionally controlled utilizing a temporal regulatory element in its promoter. Dev Biol, 2003, 259(2): 364 379[DOI]

50 Mansfield J H, Harfe B D, Nissen R, et al. MicroRNA-responsive 'sensor' transgenes uncover Hox-like and other developmentally regulated patterns of vertebrate microRNA expression. Nat Genet, 2004, 36(10): 1079 83. [DOI]

51 Ohler U, Yekta S, Lim LP, et al. Patterns of flanking sequence conservation and a characteristic upstream motif for microRNA gene identification. RNA, 2004, 10(9): 1309 1322[DOI]

52 Lai E C, Tomancak P, Williams R W, et al. Computational identification of Drosophila microRNA genes. Genome Biol, 2003, 4: R42, 1 20[DOI]

53 Legendre M, Lambert A, Gautheret D. Profile-based detection of microRNA precursors in animal genomes. Bioinformatics, 2005, 21(7): 841 845[DOI]

54 Hofacker I L. Vienna RNA secondary structure server. Nucl Acids 
Res, 2003, 31: 3429 2431[DOI]

55 Ambros V, Bartel B, Bartel D P, et al. A uniform system for microRNA annotation. RNA, 2003, 9: 277 279[DOI]

56 Griffiths-Jones S. The microRNA registry. Nucl Acids Res, 2004, 32: D109 D111[DOI]

57 Bartel D P, Chen C Z. Micromanagers of gene expression: The potentially widespread influence of metazoan microRNAs. Nat Rev Genet, 2004, 5(5): 396 400[DOI]

58 He L, Hannon G J. MicroRNAs: Small RNAs with a big role in gene regulation. Nat Rev Genet, 2004, 5(7): 522 531[DOI]

59 Hipfner D R, Weigmann K, Cohen S M. The bantam gene regulates Drosophila growth. Genetics, 2002, 161: 1527 1537

60 Brennecke J, Hipfner D R, Stark A, et al. bantam encodes a developmentally regulated microRNA that controls cell proliferation and regulates the proapoptotic gene hid in Drosophila. Cell, 2003, 113: 25 36[DOI]

61 Johnston R J, Hobert O. A microRNA controlling left/right neuronal asymmetry in Caenorhabditis elegans. Nature, 2003, 426: 845 849[DOI]

62 Chen C Z, Li L, Lodish H F, et al. MicroRNAs modulate hematopoietic lineage differentiation. Science, 2004, 303: 83 86[DOI]

$63 \mathrm{Xu}$ P, Vernooy S Y, Guo M, et al. The Drosophila microRNA mir-14 suppresses cell death and is required for normal fat metabolism. Curr Biol, 2003, 13: 790 795[DOI]

64 Chang S, Johnston R J Jr, Frokjaer-Jensen C, et al. MicroRNAs act sequentially and asymmetrically to control chemosensory laterality in the nematode. Nature, 2004, 430(7001): 785 789[DOI]

65 Yekta S, Shih I H, Bartel D P. MicroRNA-directed cleavage of HOXB8 mRNA. Science, 2004, 304(5670): 594 596[DOI]

66 Poy M N, Eliasson L, Krutzfeldt J, et al. A pancreatic islet-specific microRNA regulates insulin secretion. Nature, 2004, 432: 226 230[DOI]

67 Giraldez A J, Cinalli R M, Glasner M E, et al. MicroRNAs regulate brain morphogenesis in Zebrafish. Science, 2005, 308(5723): $833 \sim 838$ [DOI]

68 Stark A, Brennecke J, Russell R B, et al. Identification of Drosophila microRNA targets. PLOS Biol, 2003, 1: E60[DOI]

69 Enright A J, John B, Gaul U, et al. MicroRNA targets in Drosophila. Genome Biol, 2003, 5: R1[DOI]

70 Lewis B P, Shih I, Jones-Rhoades M W, et al. Prediction of mammalian microRNA targets. Cell, 2003, 115: 787 798[DOI]
71 Kiriakidou M, Nelson P T, Kouranov A, et al. A combined computational-experimental approach predicts human microRNA targets. Genes Dev, 2004, 18(10): 1165 1178[DOI]

72 Rehmsmeier M, Steffen P, Hochsmann M, et al. Fast and effective prediction of microRNA/target duplexes. RNA, 2004, 10(10): 1507 1517[DOI]

73 Krek A, Grun D, Poy M N, et al. Combinatorial microRNA target predictions. Nat Genetics, 2005, 37: 495 500[DOI]

74 John B, Enright A J, Aravin A, et al. Human microRNA Targets. Plos Biol, 2004, 2(11): e363[DOI]

75 Kuersten S, Goodwin E B. The power of the 3' UTR: Translational control and development. Nat Rev Genet, 2003, 4: 626 663[DOI]

76 Xie X, Lu J, Kulbokas E J, et al. Systematic discovery of regulatory motifs in human promoters and $3^{\prime}$ UTRs by comparison of several mammals. Nature, 2005, 434: 338 345 [DOI]

77 Lai E C. MicroRNAs are complementary to 3_UTR motifs that mediate negative post-transcriptional regulation. Nat Genet, 2002, 30: 363 364[DOI]

78 Lingel A, Simon B, Izaurralde E, et al. Structure and nucleic-acid binding of the Drosophila Argonaute 2 PAZ domain. Nature, 2003, 426: 465 469[DOI]

79 Song J J, Liu J, Tolia N H, et al. The crystal structure of the Argonaute2 PAZ domain reveals an RNA binding motif in RNAi effector complexes. Nat Struct Biol, 2003, 10: 1026 1032[DOI]

80 Yan K S, Yan S, Farooq A, et al. Structure and conserved RNA binding of the PAZ domain. Nature, 2003, 426: 468 474[DOI]

81 Lim L P, Lau N C, Garrett-Engele P, et al. Microarray analysis shows that some microRNAs downregulate large numbers of target mRNAs. Nature, 2005, 433: 769 773[DOI]

82 Ambros V. The functions of animal microRNAs. Nature, 2004, 431(7006): 350 355[DOI]

83 Takamizawa J, Konishi H, Yanagisawa K, et al. Reduced expression of the let-7 microRNAs in human lung cancers in association with shortened postoperative survival. Cancer Res, 2004, 64(11): 3753 3756

84 徐颖，朱成钢，金勇丰，等。dsRNA对家蚕核多角体病毒 (BmNPV)复制的抑制作用. 科学通报, 2004, 49(11): 1073 1078 [摘要] [PDF]

85 唐霓, 张秉强, 问歌, 等. 干扰介导对乙型肝炎病毒复制的抑制 作用. 科学通报, 2004, 49(12): 1145 1150[摘要] [PDF]

(2005-04-25 收稿, 2005-06-03 收修改稿) 